\title{
Low phospholipid-associated cholelithiasis
}

INSERM

\section{Source}

INSERM. (1999). Orphanet: an online rare disease and orphan drug data base. Low phospholipid-associated cholelithiasis. ORPHA:69663

Low phospholipid associated cholelithiasis is a rare genetic hepatic disease characterized by cholesterol gallstones and intrahepatic stones developing before the age of 40 years. 